\title{
AMENDMENTS
}

\section{Author Correction: Mammalian Atg8 proteins and the autophagy factor IRGM control mTOR and TFEB at a regulatory node critical for responses to pathogens}

Suresh Kumar, Ashish Jain (D), Seong Won Choi, Gustavo Peixoto Duarte da Silva, Lee Allers, Michal H. Mudd, Ryan Scott Peters, Jan Haug Anonsen, Tor-Erik Rusten (D), Michael Lazarou (D) and Vojo Deretic (D)

Correction to: Nature Cell Biology, https://doi.org/10.1038/s41556-020-0549-1, published online 3 August 2020

In the version of this Article originally published, some grant information was omitted from the Acknowledgements section. The last line of the section should read "T.-E.R and A.J. were supported by grants 262652 and 276070 from the Norwegian Research Council." The error has been corrected in the HTML and PDF versions of the paper.

Published online: 12 August 2020

https://doi.org/10.1038/s41556-020-0574-0

( $)$ The Author(s), under exclusive licence to Springer Nature Limited 2020

\section{Author Correction: AP-1 imprints a reversible transcriptional programme of senescent cells}

Ricardo Iván Martínez-Zamudio (D), Pierre-François Roux, José Américo N.L.F. de Freitas (D), Lucas Robinson, Gregory Doré, Bin Sun (D), Dimitri Belenki $\mathbb{D}$, Maja Milanovic (D), Utz Herbig, Clemens A. Schmitt $\mathbb{D}$, Jesús Gil $\mathbb{D}$ and Oliver Bischof (D)

Correction to: Nature Cell Biology https://doi.org/10.1038/s41556-020-0529-5, published online 8 June 2020

In the version of this Article originally published, there were several errors in Fig. 8, Extended Data Fig. 1, and source data. Mean values for CCNA2 were inadvertently copied as s.e.m. values for CCNA2 in statistical source data for Extended Data Fig. 1m. In addition, the s.e.m. values for CCNE2 were inadvertently copied as mean values for CDKN2A in statistical source data for Extended Data Fig. 1q. Corresponding Extended Data Fig. 1q was corrected accordingly to reflect the expression values of CDKN2A. Furthermore, the original statistical source data for Fig. $8 \mathrm{c}, \mathrm{d}, \mathrm{f}$ contained identical raw values and invalid integers. In addition, Irak3 expression was not displayed in the original heatmap of Fig. 8f, and the rows for CXCL5 and TGFB2 were swapped in the statistical source data file for Fig. 8d. The corrections lead to several, minor changes in the fold-change mean values that result in minimal alterations in the heatmap visualization in Fig. 8c,d,f. Additional methodology details on qPCR have also been added to the section of "Quantitative RT-qPCR" in Methods. The errors have been corrected. 





Original



Corrected

Q



Extended Data Fig. 1 | Original and Corrected.

Published online: 16 September 2020

https://doi.org/10.1038/s41556-020-00589-3

(๑) The Author(s), under exclusive licence to Springer Nature Limited 2020

\section{Publisher Correction: Recognition of RNA $N^{6}$-methyladenosine by IGF2BP proteins enhances mRNA stability and translation}

Huilin Huang, Hengyou Weng, Wenju Sun, Xi Qin, Hailing Shi, Huizhe Wu, Boxuan Simen Zhao, Ana Mesquita, Chang Liu, Celvie L. Yuan, Yueh-Chiang Hu D, Stefan Hüttelmaier, Jennifer R. Skibbe, Rui Su, Xiaolan Deng, Lei Dong, Miao Sun, Chenying Li, Sigrid Nachtergaele, Yungui Wang, Chao Hu, Kyle Ferchen, Kenneth D. Greis, Xi Jiang, Minjie Wei, Lianghu Qu, Jun-Lin Guan, Chuan He iD, Jianhua Yang (iD and Jianjun Chen (D)

Correction to: Nature Cell Biology https://doi.org/10.1038/s41556-018-0045-Z, published online 23 February 2018

In the version of this Article originally published, several equations in Fig. 3d,e were mislabelled. The correct base of all exponent equations should be 'e' instead of ' 10 ' (i.e., the equations should read as $y=\mathrm{e}^{-\mathrm{kx}}$, in which the $\mathrm{k}$ values are indicated in each figure). The errors have been corrected in the PDF and HTML versions of the paper.

Published online: 27 August 2020

https://doi.org/10.1038/s41556-020-00580-y

(C) The Author(s), under exclusive licence to Springer Nature Limited 2020 